\title{
Reliable Early Classification on Multivariate Time Series with Numerical and Categorical Attributes
}

\author{
Yu-Feng Lin ${ }^{1}$, Hsuan-Hsu Chen ${ }^{1}$, Vincent S. Tseng ${ }^{2(\bowtie)}$, and Jian Pei ${ }^{3}$ \\ ${ }^{1}$ Department of Computer Science and Information Engineering, \\ National Cheng Kung University, Tainan, Taiwan, Republic of China \\ \{aorborcord, tp6vm0vm4\} aidb.csie.ncku. edu.tw \\ ${ }^{2}$ Department of Computer Science, National Chiao Tung University, \\ Hsinchu, Taiwan, Republic of China \\ vtseng@cs.nctu.edu.tw \\ ${ }^{3}$ School of Computing Science, Simon Fraser University Burnaby, Burnaby, BC, Canada \\ jpei@cs.sfu.ca
}

\begin{abstract}
Early classification on multivariate time series has recently emerged as a novel and important topic in data mining fields with wide applications such as early detection of diseases in healthcare domains. Most of the existing studies on this topic focused only on univariate time series, while some very recent works exploring multivariate time series considered only numerical attributes and are not applicable to multivariate time series containing both of numerical and categorical attributes. In this paper, we present a novel methodology named REACT (Reliable EArly ClassificaTion), which is the first work addressing the issue of constructing an effective classifier on multivariate time series with numerical and categorical attributes in serial manner so as to guarantee stability of accuracy compared to the classifiers using full-length time series. Furthermore, we also employ the GPU parallel computing technique to develop an extended mechanism for building the early classifier efficiently. Experimental results on real datasets show that REACT significantly outperforms the state-of-the-art method in terms of accuracy and earliness, and the GPU implementation is verified to substantially enhance the efficiency by several orders of magnitudes.
\end{abstract}

Keywords: Early classification - Multivariate time series - Serial classifier · Numerical and categorical attributes $\cdot$ Shapelets $\cdot$ GPU

\section{Introduction}

Early classification, which refers to predict occurrences as early as possible, is an emerging subject in data mining with various time-sensitive applications such as health-informatics. For example, a retrospective study of clinical data from neonatal intensive unit found that abnormal heartbeat rate was significantly associated with sepsis in infants [9]. Monitoring the heartbeat time series and classifying them as early as possible may lead to earlier diagnosis and effective treatment.

The aim of early classification is naturally different than that of classic classification, which focuses only on accuracy without taking earliness into account. That is, 
early classifiers can keep similar accuracy which is comparable to classic classifiers, while they should also be able to predict the results at an earlier time. Several effective early classifiers have been proposed to make early prediction on univariate time series $[18,19]$, and these classifiers retained accuracy which was comparable to traditional classifiers $[2,4,14]$. However, to gain insights into the classification results in many applications, not only univariate time series but also multivariate time series need to be considered further.

To overcome the deficiency of the previous early classification methods that consider only univariate time series, early classification on multivariate time series has recently emerged as a novel and important topic of research [7, 8, 10]. The common idea of the existing methods is to extract multivariate shapelets as main features from all dimensions of time series with numerical attributes (or called numerical time series) that can manifest the target classes, where shapelet indicates a segment of numerical time series [8, 14, 20]. However, multivariate time series is usually composed of both numerical and categorical attributes in lots of real world data sets. For example, chronic asthmatic sufferers have to constantly observe not only vital signs and diagnostic records, but also environmental factors such as suspended particulates or humidity level. If the interactions between diagnostic records and environmental factors can be found, it is possible to predict the probability of asthmatic attack in advance using different variants of multivariate time series with numerical and categorical attributes. Moreover, Xing et al. [18, 19] argued that an early classifier should guarantee the stability of accuracy which was comparable to the classifier using full-length time series (defined as serial [18]), which can ensure an early classifier to be reliable and consistent.

In this paper, we propose a novel method for reliable early classification on Multivariate Time Series with Numerical and Categorical attributes (abbreviated as $M T S-N C$ ). However, achieving such an aim is not an easy task with the following challenges: (I) Multivariate time series is heterogeneous and each variable has different characteristics with either numerical or categorical type. Hence, it is not easy to find the potential interactions/relations between different variables in $M T S-N C$. (II) It is not an easy task to build an early classifier being serial on MTS-NC. To the best of our knowledge, the serial property is designed for shapelets extracted from multivariate time series with numerical attribute [18], and it cannot be applied directly to categorical attributes. (III) Studying the tradeoff between earliness and accuracy of $R E A C T$ on $M T S-N C$ is not an easy task. In literatures [8, 18, 19], various measurements such as discrimination, frequency, earliness are employed to estimate the qualities of features for studying the tradeoff between earliness and accuracy. However, these criteria cannot be directly applied to $M T S-N C$, and they may be ineffective in obtaining the features satisfying these conditions. (IV) The proposed classifier has to efficiently extract features on $M T S-N C$. In the feature extraction of univariate time series with categorical attribute, the existing method [2] uses a two-phase approach by generating all frequent patterns and then selecting the discriminative patterns in different phases. However, the two-phase approach cannot be directly employed to generate patterns from $M T S-N C$, which might lead to a huge number of redundant patterns. In addition, in the feature extraction of univariate/multivariate time series with numerical attribute, discovering shapelets still has a higher computation overhead on existing methods $[7,8,10]$. 
To address all of the above challenges, this paper proposes a novel framework named REACT on MTS-NC. The major contributions of this work are shown below:

1. REACT incorporates the concept of heterogeneous multivariate time series with both numerical and categorical attributes into early classification to simultaneously consider numerical and categorical time series on construction of early classifier.

2. REACT constructs a reliable early classifier which is serial and guarantees the stability of accuracy compared to the classifier using full-length time series.

3. To avoid generating a huge number of features which may be redundant, we design a procedure of feature extraction in REACT named MEG (Mining Equivalence classes with shapelet Generators) based on the concept of Equivalence Classes Mining [12, 15]. MEG can efficiently and effectively generate the discriminative features. In addition, several strategies are proposed to prune the search space and reduce the number of redundant features in the processes of feature extraction.

4. Since discovering shapelet generators takes huge calculation operations, REACT incurs still high computation overhead. In view of this, we employ and integrate concepts of GPU technique of parallel computing [4] to propose a process of parallel $M E G$ for substantially reducing the computational overhead of discovering shapelet generators.

5. We conduct an extensive empirical evaluation on several real datasets. The results show that REACT outperforms the state-of-the-art method in terms of f-score and earliness. In addition, the GPU implementation significantly runs faster than the baseline approach of building REACT by several orders of magnitudes.

The remainder of this paper is organized as follows. Section 2 introduces the background of early classification on multivariate time series. We then describe REACT in section 3. Experiments are reported in Section 4. Finally, we conclude our work and give prospective future work in Section 5.

\section{Preliminaries and Related Work}

\subsection{Preliminaries}

We introduce definitions and properties related to early classification on multivariate time series. For more details, readers can refer to [8, 14, 18, 19, 20].

Definition 1 (MTS-NC). A time series $t$ is a set of readings of the form $<r_{1}, r_{2}, \ldots$, $r_{l e n(t)}>$, where len $(t)$ is the length of $t$ and $r_{k}$ is the $k$-th reading of $t$ for all $1 \leq k \leq \operatorname{len}(t)$. Given a time series $t=\left\langle r_{1}, r_{2}, \ldots, r_{l e n(t)}\right\rangle, t$ is called categorical time series if $r_{j}$ is category for $1 \leq j \leq \operatorname{len}(t)$. On the other hand, $t$ is called numerical time series if $r_{i}$ is number for $1 \leq i \leq l e n(t)$. A $M T S-N C m t=\left\{t_{1}, t_{2}, \ldots, t_{n}\right\}$ is composed of $n$ time series, where $t_{x}$ is a categorical/numerical time series, where $1 \leq x \leq n$. Let $C(m t)$ be a corresponding class label of MTS-NC mt. Dataset of MTS-NCD is a collection of $m t$ and $C(m t)$, where $C(m t) \in$ class label set $C$. In addition, $D_{c}$ is defined the subset of $D$ carrying class label $c$, that is, $D_{c}=\{m t \mid m t \in D$ and $C(m t)=c\}$. Figure 1(a) shows an example of MTS-NC. 
Definition 2 (Subsequence and super-sequence of time series). Given two time series $t=\left\langle r_{1}, r_{2}, \ldots, r_{\text {len }(t)}\right\rangle$ and $t^{\prime}=\left\langle r_{1}{ }^{\prime}, r_{2}{ }^{\prime}, \ldots, r_{\text {len }\left(t^{\prime}\right)}{ }^{\prime}\right\rangle$, where $\operatorname{len}\left(t^{\prime}\right) \leq \operatorname{len}(t)$. We say that $t^{\prime}$ is a subsequence of time series $t$ if there exists a sequence of integers $1 \leq z_{1}$ $<z_{2}<\ldots z_{\text {len }\left(t^{\prime}\right)} \leq \operatorname{len}(t)$ such that $r_{i}^{\prime}=r_{z i}$ for all $i$, where $1 \leq i \leq \operatorname{len}\left(t^{\prime}\right)$, denoted by $t^{\prime}$ 드 $t$. On the other hand, $t$ is a super-sequence of time series $t$ '.

Definition 3 (Shapelet/Numerical feature). Given two numerical time series $n t 1$ and $n t 2=<r_{1}, r_{2}, \ldots, r_{l e n(t)}>$, where $\operatorname{len}(n t 1)=\operatorname{len}(n t 2)$, we denote the set of all distinct subsequences of time series as $S T(t)_{l}=\left\{S T(t)_{1, l}, S T(t)_{2, l}, \ldots, S T(t)_{n, l}\right\}$, and normalized Euclidean distance is defined by $\operatorname{dist}(n t 1, n t 2)=\sqrt{\frac{1}{n} \sum_{i=1}^{l e n(n t 1)}\left(n t 1_{i}-n t 2_{i}\right)^{2}}$, where $n=$ len $(t)-l+1$ and $1 \leq l<\operatorname{len}(t)$. The best matching distance is denoted by $B M D(n t 1$, $n t 2)=\operatorname{minimum}\left\{\operatorname{dist}\left(n t 1, S T(n t 2)_{j, l e n(n t 1)}\right) \mid 1 \leq j \leq \operatorname{len}(n t 2)-l+1\right\}$, where $\operatorname{len}(n t 1)=$ len(nt2). A shapelet/numerical feature is a pair $(s, \delta)$, where $\delta$ is a distance threshold, $s=<r_{1}, r_{2}, \ldots, r_{l e n(s)}>$, and $r_{i} \in \mathbb{N}$ for all $i(1 \leq i \leq \operatorname{len}(s))$. A shapelet $f$ is said to appear in a time series $t$, denoted by $f \sqsubseteq t$, if $B M D(s, t) \leq \delta$.

Definition 4 (Categorical feature). Let $c t$ be a categorical time series, a categorical feature is a subsequence of length $l$ extracted from $c t$ and denotes by $f=\left\langle r_{l}, r_{2}, \ldots, r_{l}\right\rangle$, where $l \leq l e n(c t)$.

Definition 5 (Utility of a feature). Given a feature $f$ and a dataset of MTS-NC D containing $N$ instances and $C$ different class labels, and assume that each class label $c_{i}$ has $n_{i}$ instances in $D$, where $1 \leq i \leq C$ and $N=\sum_{i=1}^{C} n_{i}$. The entropy of $D$ is defined as $E(D)$ $=-\sum_{i=1}^{C} \frac{n_{i}}{N} \log \left(\frac{n_{i}}{N}\right)$. In addition, the minimum prefix of $t$ is defined as the readings from the first reading to the $i^{\text {th }}$ reading, where $f$ firstly appears in $t$ for $1 \leq i \leq \operatorname{len}(t)$, which is denoted as minprefix $(t, f)$. Its Earliest Matching Time is the time point of minimum prefix and denoted by $\operatorname{EMT}(t, f)$. The utility of feature is defined as $U(f)=$ $\left(E(D)-E\left(D_{f}\right)\right)^{\omega} \times w \sup (f)$, where $D_{f}=\{m t \mid m t \in D, f \sqsubseteq m t\}$ is the sub-dataset of $m t$ where $f$ appears in, and $\operatorname{wsup}(f)=\frac{\sum_{f \sqsubseteq m t, m t \in \mathrm{D}} \frac{1}{E M T(m t, f)}}{|D|}$ is the weighted support to measure frequency and earliness of features, in which $|D|$ is the number of instances in $D$. The parameter $\omega \geq 1$ determines the relative importance of information versus earliness and popularity.

\begin{tabular}{|c|l|c|}
\hline SID & \multicolumn{1}{|c|}{ MiNCA } & Class \\
\hline \multirow{2}{*}{1} & $\langle\mathrm{~b}, \mathrm{c}, \mathrm{a}, \mathrm{b}, \mathrm{a}\rangle$ & \multirow{2}{*}{ low } \\
\cline { 2 - 3 } & $\langle 10,20,30,15,25\rangle$ & \\
\hline \multirow{2}{*}{2} & $\langle\mathrm{a}, \mathrm{b}, \mathrm{c}, \mathrm{c}, \mathrm{b}\rangle$ & \multirow{2}{*}{ high } \\
\cline { 2 - 3 } & $\langle 30,10,50,25,5\rangle$ & \multirow{2}{*}{} \\
\hline \multirow{2}{*}{3} & $\langle\mathrm{~b}, \mathrm{c}, \mathrm{a}, \mathrm{b}, \mathrm{a}\rangle$ & \multirow{2}{*}{ low } \\
\cline { 2 - 3 } & $\langle 15,15,30,10,20\rangle$ & \multirow{2}{*}{ high } \\
\hline \multirow{2}{*}{4} & $\langle\mathrm{~b}, \mathrm{c}, \mathrm{b}, \mathrm{b}, \mathrm{c}\rangle$ & \\
\cline { 2 - 3 } & $\langle 30,5,45,25,10\rangle$ & \\
\hline \multirow{2}{*}{5} & $\langle\mathrm{c}, \mathrm{b}, \mathrm{b}, \mathrm{a}, \mathrm{c}\rangle$ & \\
\cline { 2 - 3 } & $\langle 10,10,40,25,5\rangle$ & low \\
\hline
\end{tabular}

(a) $M T S-N C$

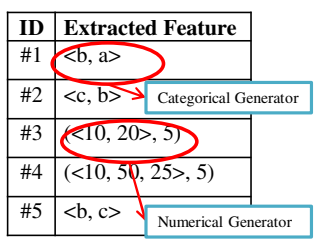

(b) Extracted Features

\begin{tabular}{|c|c|c|}
\hline SID & Encoded Sequence & Class \\
\hline 1 & $<(\# 3, \# 5)^{2},(\# 1, \# 3)^{5}>$ & low \\
\hline 2 & $<(\# 5)^{3},(\# 4)^{4},(\# 2)^{5}>$ & high \\
\hline 3 & $\left\langle(\# 3, \# 5)^{2},(\# 1, \# 3)^{5}\right\rangle$ & low \\
\hline 4 & $<(\# 5)^{2},(\# 2)^{3},(\# 4)^{4},(\# 5)^{5}>$ & high \\
\hline 5 & $<(\# 12)(\# 1, \# 4)^{4}>$ & low \\
\hline
\end{tabular}

(c) Encoding Sequence

Fig. 1. Examples of $M T S-N C$, extracted feature, and encoding sequence 
Definition 6 (Information gain and separation gap w.r.t. a shapelet). Given a dataset $D$ and a shapelet $f=(s, \delta)$, the information gain of the split point $\delta$ is defined as $I(s, \delta)=E(D)-\frac{\left|D_{f}\right|}{|D|} E\left(D_{f}\right)-\frac{\left|D_{n}\right|}{|D|} E\left(D_{n}\right)$, where $D_{f}$ is the sub-dataset of $D$ in which all instances match $f$, and $D_{n}$ is the remained time series removing $D_{f}$. The separation gap of the split point $\delta$ is computed as $G(s, \delta)=\frac{1}{\left|D_{n}\right|} \sum_{m t \in D_{n}} \operatorname{dist}(m t, s)-\frac{1}{\left|D_{f}\right|} \sum_{m t \in D_{f}} \operatorname{dist}(m t, s)$.

\subsection{Related Works}

Early classification on numerical time series aimed to classify a partial case only using the prefix of complete time series, which was first introduced by Diez et al. [4]. They simply used linear combination of available predicates of prefixes for classification. Xing et al. [18] then explored a feature based method for early classification on categorical time series. However, it had to discretize the time series when this method was applied to real-valued time series. In 2009, Xing et al. [19] proposed a novel nearest neighbor approach to tackle the problem of early classification on numeric time series. However, to gain insights into the classification results does not only be caused from univariate time series, but also multivariate time series. To overcome the deficiency, early classification on multivariate time series with numerical attribute has recently emerged as a novel and important topic of research [7, 8, 10]. In the existing frameworks, multivariate shapelets are extracted as candidates to build the early classifier, where shapelet is a segment of numerical time series $[8,14,20]$. However, multivariate time series is usually composed of numerical and categorical attributes in lots of real world data sets. Therefore, this paper simultaneously considers MTS-NC on construction of early classifier.

\section{Methodology}

In this section, we shall describe the proposed methodology named REACT (Reliable EArly ClassificaTion) on Multivariate Time Series with Numerical and Categorical attributes (abbreviated as MTS-NC). The framework of REACT is shown in Figure 2. We will introduce each process in the following subsections, and we discuss imbalance problem and implementation on GPUs in the last two subsections, respectively.

\subsection{Feature Extraction}

An equivalence class (abbreviated as EqC) was firstly introduced by Pasquier et al.[17], the maximal frequent itemsets in EqC are called closed, and the minimal frequent itemsets in $\mathrm{EqC}$ are called generators. Frequent closed patterns can form a concise and lossless representation of frequent itemsets, and they have been extensively studied [6, 13]. In addition, by Minimum Description Length principle, generators are preferable to closed patterns for model selection and classification [6, 13]. In [3, 12], authors gave discussions for the benefit of generators over closed patterns.

In the following paragraphs, we introduce how to extract categorical generators and shapelet generators from categorical and numerical time series, respectively. 


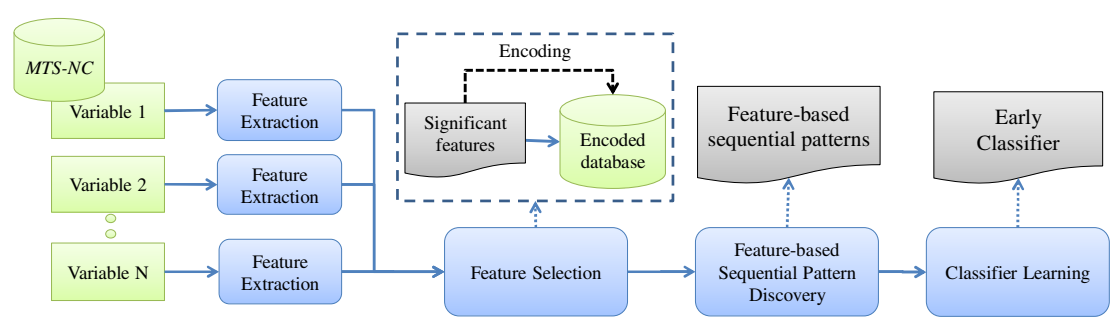

Fig. 2. The framework of REACT

\section{- Categorical Generators Extraction}

According to Definition 5, the attractive property of upper bound can be defined as below. Given a MTS-NC dataset $D$ and a categorical feature $f$, the sequential upper bound of utility is computed as $\operatorname{SeqUB}(f)=\frac{E(D)^{\omega}}{|D|} \sum_{m t \in D, f \sqsubseteq m t} \frac{1}{E M T(m t, f)+1}$. If the sequential upper bound of a categorical feature $f$ is less than min_utility, the utility of super-sequence of $f^{\prime}$ must be less than min_utility [17].

\section{Definition 7 (Extension timestamp of a categorical feature w.r.t a categorical time} series). The extension timestamp of a categorical feature $f$ w.r.t a categorical time series $S$ is defined as $E T(f)=\{t \mid t=$ matching time $+1, t \leq \operatorname{len}(S)\}$.

The essence of feature extraction of categorical generators is to check whether the patterns satisfy the stop conditions of extension or not. We first scan the projected database of prefix $s$ once, and compute the exact utility for each item $\alpha$ that the extended pattern $(s++\alpha)$ appears, where $(s++\alpha)$ defines the concatenation of $s$ and $\alpha$. Initially the prefix is empty, and the projected database of empty is the original dataset. If there are some valid items in the projected database, we then identify the equivalence class for each valid item $\alpha$ and examine whether the categorical feature $(s++\alpha)$ should be continued or not by Downward Closure Property of Non-Generator [17].

\section{- Shapelet Generators Extraction}

We adopt best matching distance as the similarity between shapelet $f$ and time series $t$ $[8,14,20]$, i.e. $B M D(f, t)$. A time series $t$ can be classified based on a shapelet $f=(s$, $\delta$ ), once we find that the distance between $t$ and $f$ is no greater than the distance threshold $\delta$. In addition, if several shapelets in the different classes satisfy the assumption, we select the first shapelet. Furthermore, to avoid existence of redundant patterns in the set of shapelets, generator mining is applied to shapelet extraction.

Definition 8 (Shapelet generator). A shapelet $f=(s, \delta)$ is called shapelet generator if (I) there is no shapelet $f^{\prime}=\left(s^{\prime}, \delta^{\prime}\right)$ satisfying $U\left(f^{\prime}\right) \geq U(f)$, (II) the covered instances of $s$ are no less than that of $s^{\prime}$, and (III) the distance between $s$ and $s^{\prime}$ is no larger than $\delta$ in the same equivalence class.

Example 1. Given two shapelets $\left.f_{1}=(<10,20\rangle, 10\right)$ and $\left.f_{2}=(<15,15,30\rangle, 10\right)$, which belong to the same equivalence class, and $f_{1}$ and $f_{2}$ have the same covered instances. In addition, $f_{1}$ has higher utility value because $f_{1}$ precedes $f_{2}$, and the distance between $<10$, $20\rangle$ and $\langle 15,15,30\rangle$ is 5 less than 10 . Therefore, we refer to $\langle 10,20\rangle$ as a subsequence of $\langle 15,15,30\rangle$, and $f_{1}$ is a generator in the equivalence class. 


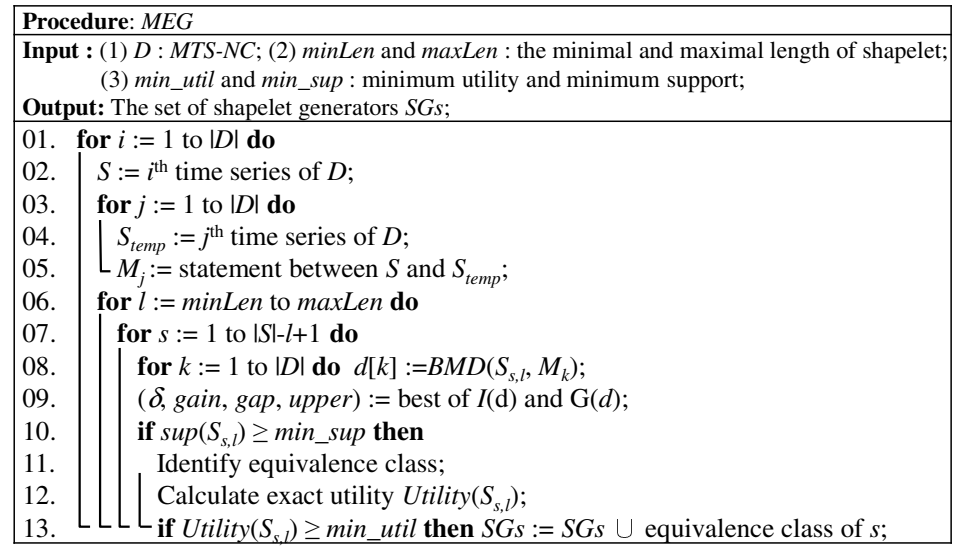

Fig. 3. Pseudo code of procedure $M E G$

We show the procedure of Mining Equivalence classes with shapelet Generators (abbreviated as $M E G$ ) in Figure 3. The procedure scans dataset $D$ once and gets distances from candidates to all time series (line 1-5). For the length of shapelet between minLen and maxLen, the best matching distance (which refers to Definition 3) are computed (line 6-8). After the distance thresholds are calculated, the information gain and separation gap are computed for each candidate (line 9), and the procedure then obtains the set of the supporting instances of shapelets to determine equivalence classes (line 10-12). If the utility of shapelet is no less than user-specified threshold, it is collected into the set $S G$ s (line 13).

\subsection{Feature Selection}

As indicated by many existing associative classification [2, 4, 14], learning an optimal set of features for classification is very expensive and non-scalable. In this work, a greedy algorithm of feature selection works as below. The procedure first ranks the equivalence classes in descending order using their utility score, and then iterates over the features starting from the highest ranked one.

Step 1. We select the feature and remove all covered instances. Here, a feature $f$ is said to cover an instance $m t$ if $m t$ matches $f$ and their classes are the same.

Step 2. We then use the next highest ranked feature to see whether it covers any of the remained instances or not.

Step 3. If it covers some of them, then we select the feature and remove all instances that are covered. Otherwise, we discard it and proceed to next one.

Step 4. This process continues step 2 to step 4 until the set of extracted features or the remaining dataset is empty. 


\subsection{Feature-Based Sequential Pattern Discovery}

In discovery of feature-based sequential patterns, we consider two kinds of combinations of features, sequential combination and simultaneous combination, to improve further the effectiveness of the classification model. Therefore, to discovery the relationship of the features mined from feature extraction, we associate each feature with a unique identifier, and then construct encoded sequence database composed of these identifiers. Figure 1(c) shows an example of encoded sequence database.

However, the encoded sequence dataset contains simultaneous event type, and there is more than one item at the same timestamp. It may increase the complexity of patter mining procedure. The downward closure Property of Feature-based Generator is thus proposed to modify for reducing the computational overhead.

Definition 9 (Simultaneous extension timestamp). The simultaneous extension timestamp of a feature-based sequential pattern $P$ for an encoded sequence $S$ is defined as $\operatorname{SET}(P, S)=\{t \mid t=$ earliest matching time in $S\}$.

Property 1 (Downward closure property of feature-based generator). Given a feature-based sequential pattern $P_{1}$, if $\exists$ a pattern $P_{2}$ such that the elements of $\left\{P_{1}\right\}$-projected database and $\left\{P_{1}\right\}$-projected database are the same, and $P_{2}$ is a sub-pattern of $P_{1}$, and then, any serial extension of $P_{1}$ are not generators. On the other hand, if $\operatorname{SET}\left(P_{1}\right)=\operatorname{SET}\left(P_{2}\right)$ for each instance and $P_{2}$ is a sub-pattern of $P_{1}$, then $P_{1}$ and all simultaneous extensions are not generators.

\subsection{Serial Decision Tree}

A tree-based classifier named $S D T$ (Serial Decision Tree) is built with all extracted information from Feature-based Sequential Pattern Discovery, as shown in Figure 4. Similar to classical decision tree algorithm [2,6,17,19], we select the attribute of the highest information gain as root of the tree, and determine the dominant class by maximizing confidence. The dataset is then divided into two sub-datasets, the first consist of the instances matching this feature and the other comprise of all remained instances. Once the root is constructed, the sub-trees of branches of the root can be constructed recursively. In addition, for each leaf node in $S D T$, we consider the stability of error rate between subspace and full-space. If the error rate of a node at time point $i$ passes the user-specified threshold $\sigma$, then the MPL of the node is set to $i+1$. We make sure that all error rates at timestamp MPL $+k$ for $k \geq 0$ are smaller than $\sigma$.

\begin{tabular}{|c|l|c|}
\hline SID & Encoding sequence & SDT $(\text { mt })^{\text {full }}$ \\
\hline 1 & $\left\langle(\# 3)^{1},(\# 8)^{2}\right\rangle$ & low \\
\hline 2 & $\left\langle(\# 1, \# 2, \# 4, \# 7)^{1},(\# 3)^{2},(\# 6)^{3}\right\rangle$ & high \\
\hline 3 & $\left.<(\# 3, \# 5)^{1},(\# 8)^{2},(\# 4, \# 7)^{3}\right\rangle$ & low \\
\hline 4 & $\left.<(\# 4, \# 7)^{1},(\# 6)^{2}\right\rangle$ & high \\
\hline 5 & $\left\langle(\# 3)^{1},(\# 6)^{2},(\# 8)^{3},(\# 7)^{4}\right\rangle$ & low \\
\hline
\end{tabular}

(a) Encoding sequence

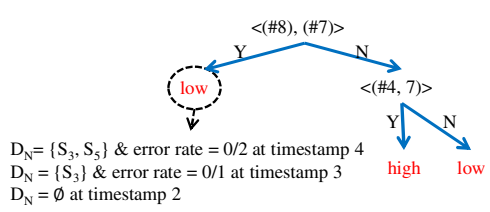

(b) Serial Decision Tree

Fig. 4. Examples of encoding sequence and serial decision tree 
Definition 10 (Error Rate of a leaf node). The error rate of a leaf node $N$ in SDT is a ratio of difference of classification results between subspace formed by the prefix of length $l$ and full-space, and is computed as $E R(N)^{l}=\frac{\mid\left\{m t \mid m t \in D_{N}, C(m t)^{l} \neq C(m t)^{f u l l_{3} \mid}\right.}{\left|D_{N}\right|}$, where $D_{N}$ is the sub-dataset which $N$ represents and $S D T(m t)$ is the class label of multivariate time series $m t$ classified by $S D T$ at time point $l$.

\subsection{Imbalance Issue}

To tackle the imbalance problem, we utilize the ratio of sub-dataset to instead of using the standard information gain and determine the discriminations of features, as shown in Definition 11.

Definition 11 (Ratio confidence and ratio entropy). Given a dataset $D$ from $C$ different classes and a sub-dataset $D_{f}$ from $D$, the ratio of $D_{f}$ for class $c$ is defined as ratio $\left(D_{f} \rightarrow c\right)=\frac{\left|\left\{m t \in D_{f} \mid c(m t)=c\right\}\right|}{|\{m t \in D \mid c(m t)=c\}|}$, where $c \in C$. The ratio confidence of class $c$ is the ability to manifest $c$, and defined as $(f \rightarrow c)=\frac{\operatorname{ratio}\left(D_{f} \rightarrow c\right)}{\operatorname{\sum ratio}\left(D_{f} \rightarrow c^{\prime}\right)}$. In addition, the ratio entropy of $D_{f}$ is computed as $r E\left(D_{f}\right)=-\sum_{c \in C} \frac{\operatorname{ratio}\left(D_{f} \rightarrow c\right)}{\sum \operatorname{ratio}\left(D_{f} \rightarrow C^{\prime}\right)} \times \log \left(\frac{\operatorname{ratio}\left(D_{f} \rightarrow c\right)}{\left.\sum \operatorname{ratio}\left(D_{f} \rightarrow c\right)^{\prime}\right)}\right)$.

Example 2. Given a pattern $P$ and a dataset of 2-classes, in which the number of instances of majority class and minority class are 1733 and 72 respectively, and suppose all instances in this dataset match $P$. The confidence and entropy of $P$ are $96 \%$ and 0.24 respectively. It is a highly discriminative pattern at first sight. As a matter of fact, this pattern always appears in this dataset. In this work, the discrimination is estimated by the modified formula such that $\operatorname{rconf}(P \rightarrow c)=50 \%$ and $r E\left(D_{P}\right)=1$.

\subsection{Implementation on GPUs}

The GPU implementation [4] employed in our study for parallel Mining Equivalence classes with shapelet Generators (abbreviated as parallel $M E G$ ) is shown in Figure 5. For each thread, parallel $M E G$ loads all subsequences of time series in dataset to shared memory $T_{i}$ and synchronizes all the threads (line 1-2). Then again, parallel $M E G$ loads all subsequences to shared memory $T_{j}$ and performs calculation of line 06-13 of algorithm $M E G$ for each thread (line 3-6). Finally, parallel $M E G$ returns the set of shapelet generators (line 7).

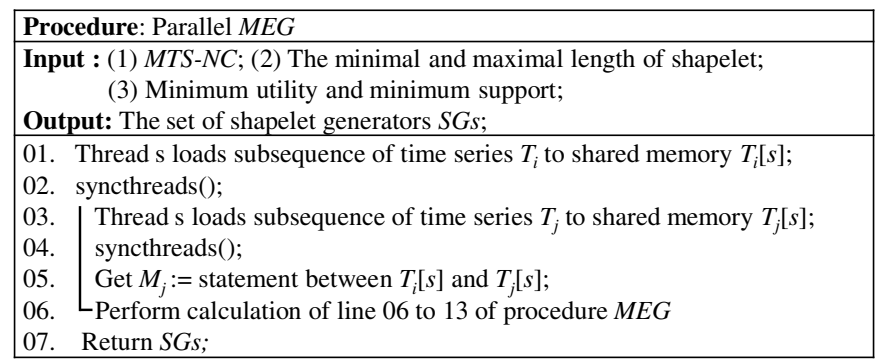

Fig. 5. Pseudo code of Algorithm Parallel $M E G$ 


\section{Experimental Evaluation}

We use several real-world datasets to evaluate the performance of the proposed classifier. All experiments were performed on a computer with a four-core Intel Xeon host CPU at $2.40 \mathrm{GHz}$ with $96 \mathrm{~GB}$ of memory, and this computer combined an NVIDIA Fermi C2075 GPU with 448 cores at $1.15 \mathrm{GHz}, 64 \mathrm{~KB}$ shared memory per GPU multiprocessor, 64KB constant memory, and 6GB global memory. All algorithms are implemented in Java language and the GPU code is implemented in CUDA C++.

The experiments were performed on several real-world datasets: drug response [3], robot execution failures [1], ECG [16], wafer [16] and asthma [11]. Table 1 shows the characteristics of the datasets in the experiments. For evaluating the performance of the proposed model, we first employ 5-fold cross validation (CV) to divide into training and testing dataset, and then generate 20 runs of 5-fold CVs to calculate the average results. We compare five versions of the algorithm named as follows: REACT, $R E A C T$-Full (REACT with full-length time series), MSD ([8], the only study addressed to early classification with interpretability on multivariate time series), MSD-Full (MSD with full-length time series), and 1NN-Full (full-length 1NN which is strongly suggested by a comparison of dozens of time series classification algorithm on various datasets [5]). The similarity measures of numerical time series for MSD and 1NN-Full are Euclidean distance.

For shapelet extraction, we set minLen $=1$ and maxLen to be $50 \%$ of the maximum length if length $>30$. Otherwise, maxLen is set to equal to the maximum length. The results are under the best parameter setting for each dataset. In the results, we report the average of f-score, applicability and earliness. The average $f$-score is computed as Avg. $f$-score $=\frac{1}{|C|} \sum_{c^{\prime} \in C} \frac{2 \times \text { precision }\left(c^{\prime}\right) \times \text { recall }\left(c^{\prime}\right)}{\operatorname{precision}\left(c^{\prime}\right)+\operatorname{recall}\left(c^{\prime}\right)}$, where Precision $\left(c^{\prime}\right)=\frac{T P}{T P+F P}$ and $\operatorname{Recall}\left(c^{\prime}\right)=$ $\frac{T P}{T P+F N}$. In this study, a true positive $(T P)$ occurs when the class of time series is predicted positive; otherwise, the model generates a false positive $(F P)$. Furthermore, a false negative $(F N)$ occurs when the model miss that the class of time series is positive.

In applicability evaluation, we regard the percentage of testing dataset which can be classified by $R E A C T$ as Applicability $(\%)=\frac{\left\{\left\{m t \mid m t \in D_{\text {testing }}, D D T(m t) \neq \varnothing\right\} \mid\right.}{\left|D_{\text {testing }}\right|}$. On the other hand, we regard the average percentage of time points used for classification as the earliness evaluation Earliness $(\%)=\frac{1}{\left|D_{\text {testing }}\right|} \sum_{m t \in D_{\text {testing }}} \frac{E M T(m t)}{\operatorname{len}(m t)}$.

Table 1. Characteristic on different datasets

\begin{tabular}{|c|c|c|c|c|c|c|}
\hline Dataset & High Dimension & Missing Value & Imbalance & Multiple Classes & Large size & Long time series \\
\hline MS70 & $\mathrm{O}$ & $\mathrm{O}$ & & & & \\
\hline Robot & & & & $\mathrm{O}$ & & \\
\hline ECG & & & & & $\mathrm{O}$ & $\mathrm{O}$ \\
\hline Wafer & & & $\mathrm{O}$ & & $\mathrm{O}$ & $\mathrm{O}$ \\
\hline Asthma & $\mathrm{O}$ & $\mathrm{O}$ & $\mathrm{O}$ & & $\mathrm{O}$ & \\
\hline
\end{tabular}


Table 2. Performances on different datasets (\%)

\begin{tabular}{|c|c|c|c|c|c|c|}
\hline & & MS70 & Robot (Avg.) & ECG & Wafer & Asthma \\
\hline \multirow{5}{*}{ Avg. f-score } & $R E A C T$ & 72.9 & 72.7 & 76.7 & 91.9 & 74.9 \\
\hline & $R E A C T$-Full & 68.5 & 70.4 & 76 & 92.1 & 69.3 \\
\hline & MSD & 60.6 & 39.6 & 58.8 & --- & --- \\
\hline & MSD-Full & 60.2 & 41.1 & 47.5 & $-\cdots$ & -- \\
\hline & 1NN-Full & 44.6 & 71.9 & 78.7 & 87.2 & 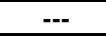 \\
\hline \multirow{5}{*}{ Applicability } & REACT & 93.8 & 94.7 & 100 & 100 & 99.4 \\
\hline & $R E A C T$-Full & 93.8 & 94.7 & 100 & 100 & 99.4 \\
\hline & MSD & 97.8 & 96.3 & 100 & --. & $--\cdot$ \\
\hline & MSD-Full & 97.8 & 96.3 & 100 & --. & -- \\
\hline & 1NN-Full & 100 & 100 & 100 & 100 & -- \\
\hline \multirow{5}{*}{ Earliness } & REACT & 22.9 & 40.7 & 10.5 & 32.8 & 73.7 \\
\hline & REACT -Full & 100 & 100 & 100 & 100 & 100 \\
\hline & MSD & 27.4 & 27.4 & 12.8 & $--\cdot$ & --- \\
\hline & MSD-Full & 100 & 100 & 100 & $-\cdots$ & $-\cdots$ \\
\hline & 1NN-Full & 100 & 100 & 100 & 100 & -- \\
\hline
\end{tabular}

Table 3. Ccomparison of computation overhead

\begin{tabular}{cccccc}
\hline Dataset & Max. Length & \#Instances & MSD $(\mathbf{s e c})$ & REACT $(\mathbf{s e c})$ & REACT-GPU(sec) \\
\hline MS70 & 5 & 53 & $62.8 \mathrm{sec}$ & 335.2 & 7.8 \\
Robot(Avg.) & 15 & 92.6 & 173.94 & 255.16 & 5.64 \\
ECG & 152 & 200 & 13168.4 & 15335.8 & 104.6 \\
Wafer & 198 & 1194 & $>2$ weeks & 150834.1 & 68228.2 \\
Asthma & 5 & 1805 & NA & 1488.1 & 370.7 \\
\hline
\end{tabular}

Table 2 lists the results on all datasets where the similarity measurement of numeric time series is Euclidean distance. The wafer dataset cannot be handled by MSD as a result of enormous computation cost. In general, REACT outperforms MSD and achieves comparable accuracy to that of $1 \mathrm{NN}-\mathrm{Full}$ because our algorithm can discover more potential information of multivariate time series. Although MSD makes the earliest classification since the criteria, namely weighted information gain, used in MSD prefers earliness and frequency rather than discrimination, the result demonstrates that it is too early to be accurate. Due to characteristic of being serial, the difference of Avg. f-score between REACT and REACT-Full is small, which shows that $R E A C T$ can capture the key features with suitable lengths of prefixes and make confident classification at appropriate timestamp.

Table 3 compares the training time of REACT, REACT on GPUs and MSD using the caching technique described in section 3. The result shows that $R E A C T$ is slower than MSD on small datasets since our approach requires feature extraction and feature-based sequential pattern discovery for each variable and class. However, on the datasets of long time series or large amount of instances, REACT is faster than MSD in execution time. The reason is that MSD have to generate a huge number of shapelet candidates and pick out a small rule set from them. In addition, REACT on GPUs runs faster than REACT over 40 to 150 orders of magnitude on MS70, Robot and ECG Datasets, and over 2 to 4 orders of magnitude on Wafer and Asthma Datasets. We observed that Wafer and Asthma Datasets are large size and Wafer is also a long time series, and they needed lots of distance calculations on subsequences of time series. 


\section{$5 \quad$ Conclusion and Future Work}

In this paper, we have proposed a novel methodology named REACT (Reliable EArly ClassificaTion) for constructing a reliable (i.e., serial) early classifier on MTS-NC. In addition, we adopt equivalence classes with generators mining to efficiently extract numerical and categorical features. Our experimental results clearly show that REACT outperforms the state-of-the-art methods in terms of accuracy and earliness. In addition, the GPU implementation significantly runs faster than the baseline approach of REACT model by several orders of magnitudes.

Although this is the first work that addresses this issue, it still leaves ample room for exploration in the future work. For example, we aim to find the significant features in different time series and use these features to build the classifier. However, the combination of non-significant features in different time series may be identifiability for early classification. In addition, the signal transform analysis techniques, e.g. wavelet or Fourier transform, may be employed to transform MTS-NC to find the significant combination features.

\section{References}

1. Bache, K., Lichman, M.: UCI machine learning repository. University of California, Irvine (2013)

2. Batal, I., Hauskrecht, M.: Constructing classification features using minimal predictive patterns. In: 10th CIKM, New York, pp. 869-878 (2010)

3. Baranzini, S.E., Mousavi, P., Rio, J., Caillier, S.J., Stillman, A., Villoslada, P., Wyatt, M.M., Comabella, M., Greller, L.D., Somogyi, R., Oksenberg, J.R.: Transcription-based prediction of response to IFN $\beta$ using supervised computational methods. PLos Biology 3(1), 166-176 (2005)

4. Chang, K.W., Deka, B., Hwu, W.M.H., Roth, D.: Efficient Pattern-Based Time Series Classification on GPU. In: ICDM, Belgium, pp. 131-140 (2012)

5. Ding, H., Trajcevski, G., Scheuermann, P., Keogh, E.: Querying and mining of time series data: experimental comparison of representations and distance measures. PVLDB 1(2) (2008)

6. Gao, C., Wang, J.: Efficient itemset generator discovery over a stream sliding window. In: 9th CIKM, Hong Kong, pp. 355-364 (2009)

7. Ghalwash, M.F., Radosavljevic, V., Obradovic, Z.: Extraction of Interpretable Multivariate Patterns for Early Diagnostics. In: 13th ICDM, Dallas, pp. 201-210 (2013)

8. Ghalwash, M.F., Obradovic, Z.: Early classification of multivariate temporal observations by extraction of interpretable shapelets. BMC Bioinformatics 13(195) (2012)

9. Griffin, M.P., Moorman, J.R.: Toward the early diagnosis of neonatal sepsis and sepsis-like illness using novel heart rate analysis. PEDIATRICS 107(1), 97-104 (2001)

10. He, G.., Duan, Y., Qian, T.Y., Chen, X.: Early prediction on imbalanced multivariate time series. In: 22th CIKM, Burlingame, pp. 1889-1892 (2013)

11. Lee, C., Chen, J.C., Tseng, V.S.: A novel data mining mechanism considering bio-signal and environmental data with application on asthma monitoring. Computer Methods and Program in Biomedicine 101(1), 44-61 (2011) 
12. Li, J., Li, H., Wong, L., Pei, J., Dong, G.: Minimum description length principle: Generators are preferable to closed patterns. In: 21 th AAAI, Boston, pp. 409-414 (2006)

13. Li, J., Liu, G., Wong, L.: Mining statistically important equivalence classes and delta-discriminative emerging patterns. In: 13th KDD, New York, pp. 430-439 (2007)

14. Lines, J., Davis, L.M., Hills, J., Bagnall, A.: A shapelet transform for time series classification. In: 18th KDD, New York, pp. 289-297 (2012)

15. Lo, D., Khoo, S., Li, J.: Mining and ranking generators of sequential patterns. In: SDM, Atlanta, pp. 553-564 (2008)

16. Olszewski, R.T.: Generalized feature extraction for structural pattern recognition in time-series data. PhD Thesis, School of Computer Science, Carnegie Mellon University, Pittsburgh (2011)

17. Pasquier, N., Bastide, Y., Taouil, R., Lakhal, L.: Discovering Frequent Closed Itemsets for Association Rules. In: Beeri, C., Bruneman, P. (eds.) ICDT 1999. LNCS, vol. 1540, pp. 398-416. Springer, Heidelberg (1998)

18. Xing, Z., Pei, J., Dong, G., Yu, P. S.: Mining sequence classifiers for early prediction. In: SDM, Atlanta, pp. 644-655 (2008)

19. Xing, Z., Pei, J., Yu, P.S.: Early classification on time series: A nearest neighbor approach. In: 21th IJCAI, Pasadena, pp. 1297-1302 (2009)

20. Ye, L., Keogh, E.: Time series shapelet: A new primitive for data mining. In: 15 th KDD, Paris, pp. 947-956 (2009) 